\title{
Repercussões para saúde mental de profissionais de enfermagem atuantes no enfrentamento à Covid-19: revisão integrativa
}

Repercussions for mental health of nursing professionals who are in the face of The Covid-19: integrative review

Repercusiones para la salud mental de los profesionales de enfermería que trabajan en el afrontamiento del Covid-19: una revisión integradora

\section{Magda Guimarães de Araujo Faria', Kalyane Cristine Ferreira Gonçalves França ${ }^{\text {II }}$, Fernanda Costa Guedes ${ }^{\mathrm{III}}$, Melissa dos Santos Soares ${ }^{\mathrm{IV}}$, Cristiane Helena Gallaschv, Luciana Valadão Vasconcelos Alves ${ }^{\mathrm{VI}}$}

\begin{abstract}
Resumo: Objetivo: identificar as repercussões na saúde mental de profissionais de enfermagem atuantes no enfrentamento da Covid-19 no primeiro ano do contexto pandêmico. Método: revisão integrativa de literatura realizada em maio de 2021 nas bases de dados eletrônicas SCOPUS, Cumulative Index to Nursing and Allied Health Literature (CINAHL), Web of Science e Literatura Latino-Americana e do Caribe em Ciências da Saúde (LILACS). Resultados: foram encontradas 1249 publicações cuja aplicação de critérios de inclusão, exclusão e leitura crítica, culminou na análise de 18 artigos provenientes de vivências da China, Turquia, Itália, Canadá, Estados Unidos, Filipinas, Brasil, Portugal e Etiópia. As principais repercussões envolvidas no adoecimento mental foram: ansiedade, depressão, estresse, síndrome de Burnout, transtornos mentais do sono e transtorno de estresse pós-traumático. Conclusão: a saúde mental dos enfermeiros encontra-se extremamente fragilizada frente ao contexto pandêmico. Essa emergência na saúde mundial, acabou por desencadear consequências físicas, emocionais e psicológicas nesses trabalhadores.
\end{abstract}

Descritores: Saúde do trabalhador; Saúde mental; Enfermeiras e enfermeiros; Infecções por coronavírus; Pandemias

Abstract: Objective: To identify the repercussions on mental health of nursing professionals who are in the face of Covid-19 in the first year of the pandemic context. Method: integrative literature review conducted in May 2021 in the

\footnotetext{
${ }^{\text {I }}$ Doutora em enfermagem. Universidade do Estado do Rio de Janeiro. Faculdade de Enfermagem. Rio de Janeiro, RJ, Brasil. E-mail: magda.faria@live.com. Orcid: 0000-0001-9928-6392

II Estudante de Enfermagem. Universidade do Estado do Rio de Janeiro. Faculdade de Enfermagem. Rio de Janeiro, RJ, Brasil. E-mail: kaly.franca@gmail.com Orcid: 0000-0001-6711-9557

III Estudante de Enfermagem. Universidade do Estado do Rio de Janeiro. Faculdade de Enfermagem. Rio de Janeiro, RJ, Brasil. E-mail: fesu.guedes@gmail.com Orcid: 0000-0002-8050-3833

IV Estudante de Enfermagem. Universidade do Estado do Rio de Janeiro. Faculdade de Enfermagem. Rio de Janeiro, RJ, Brasil. E-mail: melhilario100@gmail.com Orcid: 0000-0002-4717-6092

v Doutora em enfermagem. Universidade do Estado do Rio de Janeiro. Faculdade de Enfermagem. Rio de Janeiro, RJ, Brasil. E-mail: cristiane.gallasch@gmail.com. Orcid: 0000-0002-0823-0818

${ }^{\mathrm{VI}}$ Doutora em enfermagem. Instituto Federal de Educação, Ciência e Tecnologia Fluminense. Macaé, RJ, Brasil. E-mail: lucianavvalves@hotmail.com. Orcid: 0000-0003-2159-543X
} 
Repercussões para saúde mental de profissionais de enfermagem atuantes no enfren.... $\mid 2$

electronic databases SCOPUS, Cumulative Index to Nursing and Allied Health Literature (CINAHL), Web of Science and Latin American and Caribbean Literature on Health Sciences (LILACS). Results: 1,249 publications were found whose application of inclusion, exclusion and critical reading criteria culminated in the analysis of 18 articles from experiences from China, Turkey, Italy, Canada, United States, Philippines, Brazil, Portugal and Ethiopia. The main repercussions involved in mental illness were anxiety, depression, stress, Burnout syndrome, mental sleep disorders and posttraumatic stress disorder. Conclusion: The mental health of nurses is extremely fragile in the face of the pandemic context. This emergency in world health eventually triggered physical, emotional and psychological consequences in these workers.

Descriptors: Occupational health; Mental health; Nurses; Coronavirus infections; Pandemics

Resumen: Objetivo: identificar las repercusiones en la salud mental de los profesionales de enfermería que trabajan en el afrontamiento del Covid-19 en el primer año del contexto pandémico. Método: revisión integrativa de la literatura realizada en mayo de 2021 en las bases de datos electrónicas SCOPUS, Índice Acumulativo de Literatura de Enfermería y Afines en Salud (CINAHL), Web of Science y Literatura de Ciencias de la Salud de América Latina y el Caribe (LILACS). Resultados: se encontraron 1249 publicaciones cuya aplicación de criterios de inclusión, exclusión y lectura crítica culminó en el análisis de 18 artículos de experiencias en China, Turquía, Italia, Canadá, Estados Unidos, Filipinas, Brasil, Portugal y Etiopía. Las principales repercusiones de la enfermedad mental fueron: ansiedad, depresión, estrés, síndrome de Burnout, trastornos del sueño mental y trastorno por estrés postraumático. Conclusión: la salud mental de las enfermeras es extremadamente frágil frente al contexto pandémico. Esta emergencia sanitaria mundial acabó provocando consecuencias físicas, emocionales y psicológicas para estos trabajadores.

Descriptores: Salud laboral; Salud mental; Enfermeras y enfermeros; Infecciones por coronavírus; Pandemias

\section{Introdução}

O dia 11 de março de 2020 foi marcado pela divulgação da primeira pandemia da história provocada por um coronavírus, demarcando a disseminação mundial da SARS-CoV-2, comumente chamada Covid-19. ${ }^{1}$ Passado o primeiro ano de pandemia, o cenário atual brasileiro aponta para um quadro ainda mais caótico, já que o número de mortes por Covid-19 até o dia 16 de maio de 2021 indica o quantitativo de mais de 435 mil, as quais mais de 153 mil foram registradas apenas nos últimos dois meses. ${ }^{2}$

O trabalho dos profissionais de saúde na linha de frente do combate à doença potencializa a vulnerabilidade em relação ao contágio, ${ }^{3}$ e no cenário mundial a contaminação destes é uma realidade. $\mathrm{Na}$ China, país que relatou os primeiros casos da doença, foram 23 profissionais mortos. ${ }^{4}$ Apesar da intensa e acelerada modificação nos perfis de incidência e mortalidade, observa-se que a realidade brasileira possui dados bem diferentes das estatísticas chinesas, já que em maio de 2021 apenas na categoria de enfermagem, já são quase 56 mil casos confirmados e 778 mortes. ${ }^{5}$ 
3 | Faria MGA, França KCFG, Guedes FC, Soares MS, Gallasch CH, Alves LVV

Neste contexto, observa-se que a realidade global bem como a possibilidade de contaminação gera um impacto na saúde mental dos profissionais da saúde, sobretudo dos enfermeiros atuantes na assistência direta ao usuário, afetando a condição de bem-estar e na capacidade de execução de suas atividades. ${ }^{6}$ Ademais, as incessantes instruções sobre condutas pessoais no contexto da pandemia, aliadas à escassez dos materiais de proteção relatada no início da emergência sanitária, além do contato rotineiro com situações de adoecimento e morte, podem desencadear a estes profissionais inúmeros quadros de sofrimento psíquico. ${ }^{7}$

Ademais, ressalta-se que o contexto pandêmico evidentemente trouxe modificações na práxis dos profissionais de enfermagem, gerando desconforto, medo e sensações ainda pouco exploradas que culminam em repercussões para saúde mental. Neste sentido, identificar tais repercussões são essenciais para a construção de estratégias de enfrentamento, apoio e fortalecimento destes profissionais.

A relevância desta pesquisa está associada à necessidade de ressaltar o aspecto humano dos profissionais de saúde, que devido a fragilidade emocional ocasionada pelo contexto de crise pode culminar em processos de adoecimento, afetando a saúde, o convívio social, o núcleo familiar e seu processo de trabalho. Sabendo da repercussão na saúde mental destes profissionais, em especial da enfermagem, o objetivo deste artigo é identificar as repercussões na saúde mental de profissionais de enfermagem atuantes no enfrentamento da Covid-19 no primeiro ano do contexto pandêmico.

\section{Método}

Trata-se de uma pesquisa do tipo revisão integrativa de literatura (RIL), que leva em conta o agrupamento de produções científicas voltadas para um determinado assunto, de forma a sistematizar diversos estudos já publicados e sintetizá-los em uma nova obra. ${ }^{8}$ Por se tratar de uma análise de informações já publicizadas e pela ausência de qualquer tipo de coleta de dados com seres humanos, tornou-se dispensável a apreciação ética desta investigação.

Foram cumpridos os seis passos da RIL, a citar: 1. delineamento da pergunta norteadora; 2. 
Repercussões para saúde mental de profissionais de enfermagem atuantes no enfren.... $\mid 4$ Estabelecimento dos critérios de inclusão, exclusão e estratégias de busca; 3. definição das informações que serão extraídas e analisadas dos estudos elencados; 4. Avaliação dos manuscritos; 5. interpretação dos resultados; 6. apresentação da síntese dos resultados..$^{8-9}$

Primeiramente, selecionou-se a temática e delineou-se a pergunta com o emprego da estratégia PICo (População, fenômeno de Interesse e Contexto). ${ }^{8,10}$ Definiu-se como População (P) os profissionais de enfermagem, para o Fenômeno de Interesse (I), as repercussões para a saúde mental no primeiro ano pandêmico e para o Contexto (Co), o cenário de pandemia da Covid-19. Assim, obteve-se a questão de revisão: Quais são as repercussões na saúde mental dos profissionais de enfermagem atuantes no enfrentamento à Covid-19 no primeiro ano do contexto pandêmico?

No segundo passo foram estabelecidos os critérios de elegibiliade, considerou-se para inclusão: publicações no formato artigo científico, publicados em português, inglês ou espanhol com o uso de restrição temporal referente aos anos 2020-2021. ${ }^{8}$ Foram considerados critérios de exclusão: revisões de literatura, manuscritos teóricos ou reflexivos, investigações cuja coleta de dados se deu antes do reconhecimento da pandemia e aqueles que não contemplavam a pergunta de pesquisa, ou seja, impertinência temática. Vale salientar que os manuscritos duplicados foram computados uma única vez.

Para esta busca, optou-se pela utilização de descritores nas bases de dados eletrônicas SCOPUS, Cumulative Index to Nursing and Allied Health Literature (CINAHL), Web of Science e Literatura Latino-Americana e do Caribe em Ciências da Saúde (LILACS). Os descritores escolhidos e pesquisados por seus equivalentes em inglês, de acordo com os termos do Medical Subject Headings. O levantamento foi realizado em maio de 2021 de modo com os termos: ((Nursing) OR (Nurse) OR (Nursing, team)) AND (Mental Health) AND ((Covid-19) OR (SARS-CoV2)).

Para o terceiro passo, definiu-se as informações que seriam extraídas e analisadas dos estudos elencados, a citar: título do artigo, país de publicação, ano de publicação, objetivo do estudo e repercussões para a saúde mental. ${ }^{8}$ 
5 | Faria MGA, França KCFG, Guedes FC, Soares MS, Gallasch CH, Alves LVV

O quarto passo da RIL refere-se a análise dos estudos. O fluxograma com a representação de elegibilidade e inclusão de artigos na seleção dos estudos está disposto na Figura 1.

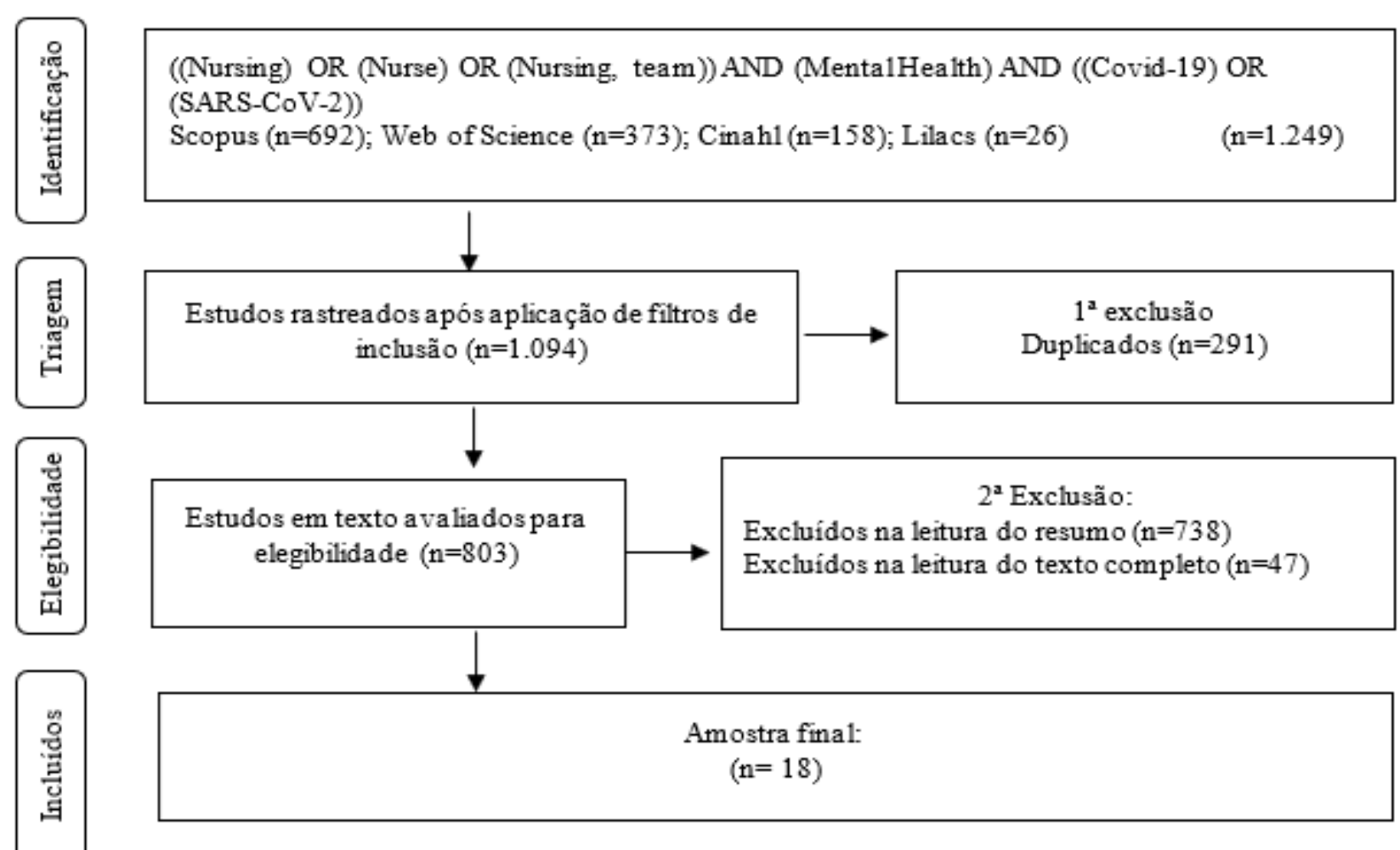

Figura 1 - Fluxograma com representação de elegibilidade e inclusão de artigos na seleção dos estudos

No quinto passo foi realizada a análise dos estudos de forma descritiva e interpretativa, considerando os aspectos éticos, respeitando a autoria das ideias, os conceitos e as definições apontadas pelos autores. A avaliação dos estudos relevantes concentrou-se no administrador de referências denominado Rayyan ${ }^{11}$ com a colaboração de três avaliadores independentes.

A sexta etapa da RIL é a apresentação da revisão em formato de quadro sinóptico para sintetizar os achados. ${ }^{8}$ As variáveis para compor o quadro foram as mesmas utilizadas na etapa três da RIL: amostra e local do estudo, objetivo e repercussões para saúde mental.

\section{Resultados}

Foram encontradas 1.249 publicações, cuja aplicação de filtros, exclusão de duplicatas e leitura completa gerou uma amostra de 18 artigos, ${ }^{12-29}$ conforme descrito no Quadro 1.

Quadro 1. Síntese dos artigos incluídos no estudo - Brasil, 2021 
Repercussões para saúde mental de profissionais de enfermagem atuantes no enfren.... $\mid 6$

\begin{tabular}{|c|c|c|}
\hline $\begin{array}{l}\text { Amostra, período } \\
\text { de coleta e local do } \\
\text { estudo }\end{array}$ & Objetivo & $\begin{array}{l}\text { Repercussões na Saúde } \\
\text { Mental }\end{array}$ \\
\hline $\begin{array}{l}180 \text { enfermeiros, } \\
\text { março-abril } 2020 \\
\text { China }^{12}\end{array}$ & $\begin{array}{l}\text { Investigar os papéis mediadores do afeto positivo e negativo na relação } \\
\text { entre resiliência e burnout em hospitais de Wuhan no pico da } \\
\text { pandemia COVID-19 }\end{array}$ & Burnout \\
\hline $\begin{array}{l}169 \text { enfermeiros, } \\
\text { abril-agosto, } 2020 \text {, } \\
\text { Turquia }^{13}\end{array}$ & $\begin{array}{c}\text { Examinar o estresse percebido e os fatores que afetam relacionados à } \\
\text { pandemia COVID-19 de enfermeiras de emergência no primeiro } \\
\text { estágio da pandemia }\end{array}$ & Estresse \\
\hline $\begin{array}{l}1005 \text { enfermeiros, } \\
\text { fevereiro-abril, } \\
\text { 2020, } \text { Itália }^{14}\end{array}$ & $\begin{array}{l}\text { Avaliar a prevalência de ansiedade, distúrbios do sono e autoeficácia e } \\
\text { seus fatores preditivos entre enfermeiros que enfrentam o COVID -19 }\end{array}$ & $\begin{array}{l}\text { Ansiedade e transtornos } \\
\text { mentais do sono }\end{array}$ \\
\hline $\begin{array}{l}270 \text { enfermeiros, } \\
\text { maio, } 2020 \\
\text { Turquia }^{15}\end{array}$ & $\begin{array}{l}\text { Determinar os níveis de ansiedade dos enfermeiros causados pelas } \\
\text { mudanças vivenciadas durante a pandemia de Covid-19 }\end{array}$ & Ansiedade \\
\hline $\begin{array}{l}3676 \text { enfermeiros, } \\
\text { junho-julho, } 2020 \text {, } \\
\text { Canadá }^{16}\end{array}$ & $\begin{array}{c}\text { Examinar o impacto das condições de trabalho do COVID-19 nos } \\
\text { resultados de saúde mental dos enfermeiros }\end{array}$ & $\begin{array}{l}\text { Transtorno de estresse } \\
\text { pós-traumático, } \\
\text { ansiedade, depressão }\end{array}$ \\
\hline $\begin{array}{l}587 \text { enfermeiros, } \\
\text { maio-junho, 2020, } \\
\text { EUA }^{17}\end{array}$ & $\begin{array}{l}\text { Descrever os níveis de insônia, fadiga e bem-estar psicológico } \\
\text { relacionadas às equipes de enfermagem durante a pandemia de } \\
\text { COVID-19 nos Estados Unidos }\end{array}$ & $\begin{array}{l}\text { Transtornos mentais do } \\
\text { sono, transtorno de } \\
\text { estresse pós-traumático, } \\
\text { ansiedade e depressão }\end{array}$ \\
\hline $\begin{array}{l}291 \text { enfermeiros, } \\
\text { março-abril, 2020, } \\
\text { Itália }^{18}\end{array}$ & $\begin{array}{l}\text { Avaliar a síndrome de Burnout em enfermeiros que prestam } \\
\text { assistência a pacientes com doença por coronavírus }\end{array}$ & Burnout \\
\hline $\begin{array}{l}12596 \text { enfermeiros, } \\
\text { março-abril, 2020, } \\
\text { China }^{19}\end{array}$ & $\begin{array}{l}\text { Avaliar trauma, Burnout e transtorno de estresse pós-traumático entre } \\
\text { enfermeiras na pandemia de COVID-19 }\end{array}$ & $\begin{array}{l}\text { Transtorno de estresse } \\
\text { pós-traumático, Burnout }\end{array}$ \\
\hline $\begin{array}{l}325 \text { enfermeiros, } \\
\text { abril-maio, } 2020 \\
\quad \text { Filipinas }^{20}\end{array}$ & $\begin{array}{l}\text { Identificar a influência relativa da resiliência pessoal, suporte social e } \\
\text { suporte organizacional na redução da ansiedade entre enfermeiros } \\
\text { atuantes na linha de frente }\end{array}$ & Ansiedade \\
\hline $\begin{array}{l}123 \text { enfermeiros, } \\
\text { abril, 2020, } \\
\text { Turquia }^{21}\end{array}$ & $\begin{array}{l}\text { Investigar a ansiedade relacionada à pandemia em enfermeiras que } \\
\text { trabalham em um hospital universitário na Turquia }\end{array}$ & Ansiedade \\
\hline $\begin{array}{l}713 \text { enfermeiros, } \\
\text { maio-julho, } 2020 \\
\text { Turquia }^{22}\end{array}$ & $\begin{array}{l}\text { Determinar os níveis de estresse, depressão e Burnout de enfermeiras } \\
\text { da linha de frente durante a pandemia de COVID-19 }\end{array}$ & $\begin{array}{l}\text { Estresse, depressão e } \\
\text { Burnout }\end{array}$ \\
\hline
\end{tabular}


7 | Faria MGA, França KCFG, Guedes FC, Soares MS, Gallasch CH, Alves LVV

\begin{tabular}{|c|c|c|}
\hline $\begin{array}{l}1794 \text { enfermeiros, } \\
\text { março, } 2020 \\
\text { China }^{23}\end{array}$ & $\begin{array}{l}\text { Investigar a prevalência de insônia entre enfermeiras da linha de frente } \\
\text { que lutam contra COVID-19 em Wuhan }\end{array}$ & $\begin{array}{l}\text { Transtornos mentais do } \\
\text { sono }\end{array}$ \\
\hline $\begin{array}{l}388 \text { profissionais } \\
\text { de enfermagem, } \\
\text { março-abril, 2020, } \\
\text { Brasil }^{24}\end{array}$ & $\begin{array}{l}\text { Identificar a prevalência e fatores associados à ansiedade e depressão } \\
\text { em profissionais de enfermagem que atuam no enfrentamento da } \\
\text { COVID-19 em hospital universitário }\end{array}$ & Ansiedade e depressão \\
\hline $\begin{array}{l}767 \text { enfermeiros, } \\
\text { março-abril, } 2020 \\
\text { Portugal }^{25}\end{array}$ & $\begin{array}{l}\text { Descrever o estado de saúde mental dos enfermeiros durante o surto de } \\
\qquad \text { Covid-19 }\end{array}$ & $\begin{array}{c}\text { Depressão, ansiedade e } \\
\text { estresse }\end{array}$ \\
\hline $\begin{array}{l}320 \text { enfermeiros, } \\
\text { abril-maio, } 2020 \\
\text { EUA }^{26}\end{array}$ & $\begin{array}{l}\text { Examinar o impacto de vários fatores que afetam a saúde mental dos } \\
\text { enfermeiros durante a pandemia COVID-19 }\end{array}$ & $\begin{array}{c}\text { Depressão, ansiedade e } \\
\text { estresse }\end{array}$ \\
\hline $\begin{array}{l}1457 \text { enfermeiros, } \\
\text { abril-maio, } 2020 \text {, } \\
\text { Turquia }^{27}\end{array}$ & $\begin{array}{l}\text { Investigar os níveis de ansiedade de enfermeiros que prestam cuidados } \\
\qquad \text { a pacientes com COVID-19 na Turquia }\end{array}$ & Ansiedade \\
\hline $\begin{array}{l}109 \text { enfermeiros, } \\
\text { maio, } 2020 \\
\text { Canadá }^{28}\end{array}$ & $\begin{array}{l}\text { Examinar a saúde mental os enfermeiros que prestam atendimento } \\
\text { direto ao paciente durante a fase inicial da pandemia COVID-19 no } \\
\text { Canadá }\end{array}$ & $\begin{array}{l}\text { Transtorno de estresse } \\
\text { pós-traumático, } \\
\text { ansiedade, depressão, } \\
\text { estresse }\end{array}$ \\
\hline $\begin{array}{l}302 \text { enfermeiros, } \\
\text { setembro-outubro, } \\
2020 \text { Etiópia }^{29}\end{array}$ & $\begin{array}{l}\text { Avaliar a prevalência e os fatores associados de ansiedade, depressão e } \\
\text { estresse entre enfermeiros que trabalham em hospitais de referência } \\
\text { do noroeste de Amhara }\end{array}$ & $\begin{array}{c}\text { Depressão, ansiedade e } \\
\text { estresse }\end{array}$ \\
\hline
\end{tabular}

Ressalta-se que os estudos analisados foram investigações de natureza quantitativa e descritiva, classificados como nível de evidência 4, ou seja, são estudo com delineamento não experimental como pesquisas descritivas correlacionais e/ou qualitativa como estudos de caso. ${ }^{30}$ Observou-se a existência de seis repercussões para a saúde mental dos profissionais de enfermagem atuantes na linha de frente do enfrentamento à Covid-19, a citar: ansiedade, depressão, estresse, transtorno de estresse pós-traumático, transtornos mentais do sono e Burnout.

\section{Discussão}

As respostas emocionais à emergência de saúde pública trazidas pela disseminação mundial 
Repercussões para saúde mental de profissionais de enfermagem atuantes no enfren.... $\mid 8$ do vírus SARS-CoV-2 estão associadas aos mecanismos de enfrentamento individuais e coletivos, influenciando o modo de agir de toda a população, principalmente os profissionais de saúde. ${ }^{31}$

Dessa forma, a partir dos estudos coletados, notou-se que a ansiedade é a repercussão para saúde mental mais referida entre os profissionais de enfermagem atuantes no enfrentamento a Covid-19. ${ }^{14-17,20-21,24-29} \mathrm{O}$ sentimento de ansiedade é observado com certa frequência no cotidiano de profissionais de saúde, entretanto, a aproximação de situações como a morte iminente de pacientes pode apontar para existência de níveis de ansiedade até $40 \%$ maiores do que os normais. ${ }^{32}$

O levantamento realizado apontou a prevalência de ansiedade variando entre $48 \%{ }^{24}$ a $86 \%,{ }^{15-16}$ sendo observado em um dos estudos, a existência de ansiedade disfuncional em quase $40 \%$ da amostra. ${ }^{20}$ É importante ressaltar que a prevalência de ansiedade entre profissionais de enfermagem é superior aos índices observados em outras categorias da saúde, como em relação a fisioterapia com taxa de $32 \%{ }^{33}$ e medicina com prevalência de $29,7 \%{ }^{34}$

Apesar da assistência aos usuários com Covid-19 já representar um grande motivo de ansiedade, os estudos indicam ainda outras gêneses como a exposição da família, ${ }^{28}$ preocupações sobre quantidade de Equipamento de Proteção Individual (EPI) disponível, ${ }^{25}$ mudanças de protocolo de atendimento, levando a repentinas modificações no processo de trabalho. ${ }^{16}$

Observou-se a existência de características relacionadas a alta prevalência de ansiedade, a citar: 1 Ser do sexo feminino, ${ }^{27}$ estar na assistência direta ao usuário infectado ${ }^{26}$ e, experiência na profissão que pode ser um fator determinante na percepção da vulnerabilidade vivenciada. ${ }^{21}$

É importante enfatizar que é percebida uma elevação dos níveis de ansiedade entre enfermeiros da linha de frente ao combate à Covid-19, sobretudo após a identificação do cenário pandêmico. Em pesquisas realizadas em fevereiro de 2020, ou seja, anteriores a identificação da pandemia, os índices de sinais de ansiedade entre enfermeiros variou entre $8 \%{ }^{35}$ e $31 \%{ }^{36}$

A depressão também foi considerada uma temática prevalente entre os profissionais atuantes no enfrentamento à Covid-19. 16,22,24-26,28-29 É válido ressaltar que os índices de acometimento dos profissionais de enfermagem atuantes na linha de frente pela depressão são flutuantes variando 
entre $26 \%{ }^{26}$ a $55 \%$ da amostra. ${ }^{28,29}$ Trata-se de uma patologia grave que atinge negativamente como o indivíduo pensa, sente e age, causando sentimento de tristeza e desinteresse sobre as atividades do dia a dia, afetando grupos funcionais do corpo, tais como: cognitivo, motivacional, somático, afetivo, comportamental e vegetativo, além da capacidade laboral do trabalhador. ${ }^{37}$

Observou-se a tendência de níveis mais elevados de depressão entre enfermeiros com doença crônica, cuja prevalência foi superior a cinco vezes quando comparada a grupos sem esse tipo de enfermidade. O histórico de transtorno mental prévio também elevou o risco de depressão, chegando a índices até sete vezes superiores quando comparados aos demais enfermeiros. ${ }^{29}$

Outro fator preponderante para o acometimento por depressão foi a idade dos enfermeiros, já que quanto mais jovens e com menos experiência, maior era o risco de acometimento por sintomas depressivos. ${ }^{22}$ Não obstante, o número de horas trabalhadas e o trabalho deslocado para outros setores também foram observados como importantes fatores de risco. ${ }^{25}$

Paradoxalmente ao aspecto expositivo inerente ao trabalho de enfermagem na linha de frente, o isolamento social recomendado como estratégia de contenção da pandemia foi associado ao aumento da prevalência de depressão. ${ }^{26}$

Ressalta-se que o isolamento social foi determinante em processos de adoecimento mental em toda a população, não apenas de profissionais de saúde. Em relação aos adultos jovens, comparativos entre janeiro de 2020 e maio de 2020 apontou-se aumento estatístico na prevalência de sintomas depressivos. ${ }^{38}$ Desse modo, o isolamento social relacionado a população idosa está relacionado ao aumento de casos de depressão, configurando-se um problema de saúde pública. ${ }^{39}$

Outra repercussão evidenciada foi o estresse. Esse, em contraste com as patologias anteriormente citadas, não é definido por ser uma doença propriamente dita, mas sim como uma reação do corpo a algum tipo de mudança, podendo servir de estopim para desencadear outros problemas psicológicos ${ }^{13,22,25-26,28-29,40}$

A taxa de prevalência de estresse observada nos estudos analisados variou de $20 \%$ a $45 \%$ da amostra. ${ }^{29}$ Novamente, ser do sexo feminino, jovem e com poucos anos de formação foram 
reconhecidos como importantes fatores de risco. ${ }^{13}$ Além disso, enfermeiros deslocados para novos setores, sobretudo para cuidados intensivos à vítimas de Covid-19, foram preponderantes no registro de estresse ocupacional, assim como o trabalho noturno. ${ }^{25-26,29}$

Em tempos pandêmicos, a literatura relata como situações de estresse ocupacional entre os profissionais de saúde: a falta de EPIs, o possível contágio diante da doença, o receio de transmiti-la para os familiares e o constante questionamento sobre o próprio desempenho. ${ }^{41-42}$ Além disso, a sensação de incapacidade dos profissionais em lidar com as próprias demandas psicológicas, gerando a necessidade premente de apoio, nem sempre atendida, o que por sua vez, eleva o risco de adoecimento, sendo então emergentes as ações de gestão de segurança para a saúde mental. ${ }^{43-44}$

A evolução gradual das situações de estresse aliada a outros fenômenos pode gerar a agravação do quadro psíquico, culminando na Síndrome de Burnout, a qual o esgotamento, a angústia e a desesperança são sinais recorrentes, sobretudo aliadas ao contexto do enfrentamento à Covid-19. 12,18-19,22 Acrescenta-se ainda que o processo de trabalho dos profissionais de saúde traduzse em potencial risco de desenvolvimento da síndrome de Burnout. ${ }^{45}$

Assim sendo, a prevalência de Burnout relatada na literatura alcançou índices superiores a $50 \%$ da amostra. ${ }^{12} \mathrm{O}$ sexo feminino e a recente formação foram fatores de risco para aumento da prevalência, entretanto, no que diz respeito às dimensões específicas da Síndrome de Burnout, apenas a exaustão emocional apresentou diferenças significativas, estando a despersonalização e a realização pessoal equiparadas quanto ao sexo dos participantes. ${ }^{18}$

Enfermeiros atuantes em emergências, cuidados intensivos ou enfermarias Covid possuíam índice superior de prevalência de Burnout quando comparados aos profissionais de outros setores do hospital. Entre os acometidos, sensações de desesperança, memórias infelizes e irritabilidade eram constantes. ${ }^{19}$ Profissionais atuantes na linha de frente que positivaram em testes para Covid19 e foram afastados das funções laborais, também apresentaram elevados índices de Burnout após o retorno ao trabalho. ${ }^{22}$

É necessário enfatizar a estreita relação entre o Burnout, sobrecarga e as condições de 
11 | Faria MGA, França KCFG, Guedes FC, Soares MS, Gallasch CH, Alves LVV

trabalho, o que geral alterações fisiológicas e psicológicas na saúde dos trabalhadores, atingindo de forma significativa o desempenho de suas atividades. ${ }^{46}$

Outra repercussão importante na saúde mental de enfermeiros da linha de frente do cuidado, são os transtornos mentais relacionados ao sono. ${ }^{14,22-23}$ O sono é primordial para o bom funcionamento físico, cognitivo, ocupacional e social do indivíduo e os profissionais de saúde possuem corriqueiramente problemas associados ao padrão de sono, sobretudo pelo ritmo de trabalho. ${ }^{47}$ Os índices de alterações do padrão do sono percebidos entre enfermeiros da linha de frente variaram entre $53 \%{ }^{23}$ a $87 \% .^{17}$

Ao atuar na linha de frente ao cuidado de enfermagem para usuários com Covid-19, observou-se a existência de perfis protetivos a ocorrência de transtornos mentais do sono, como ser do sexo masculino e a atuação na gestão, educação ou pesquisa e o controle da ansiedade, já que está relacionada ao aumento da prevalência de insônia. ${ }^{14}$

Acrescenta-se ainda que neste primeiro ano de enfrentamento à pandemia foram observadas sintomatologias condizentes a outros transtornos, como o transtorno de estresse pós-traumático. ${ }^{16-}$ ${ }^{17,19,28}$ A prevalência deste transtorno variou entre $38 \%{ }^{28}$ e $55 \% .{ }^{17}$ chegando ao acometimento de $56 \%$ da amostra. ${ }^{28}$ Apesar do alto percentual de acometimento, ainda são escassos os relatos, os quais acredita-se que haverá um incremento quantitativo nos próximos meses.

Apesar da atuação na área da enfermagem não ser apontada como fator de risco para desenvolvimento de transtorno de estresse pós-traumático, observa-se um padrão próximo a realidade demográfica da enfermagem, já que ser mulher, trabalhar em hospital e preocupar-se em possíveis contaminações são condições relacionadas ao aumento dos índices deste transtorno. ${ }^{48}$

É válido ressaltar que a associação de todas as repercussões para a saúde mental quando avaliadas em coletivo e associadas a fatores como sobrecarga de trabalho e autonomia insuficiente, podem culminar em ideação suicida. ${ }^{49} \mathrm{O}$ fenômeno do suicídio associado a atuação na linha de frente do Covid-19 ainda é pouco estudado e carece uma meticulosa avaliação, sobretudo pela conjunção de vários fatores de risco apresentados pelos enfermeiros, contudo, ressalta-se que 
avaliações preliminares ao contexto pandêmico aponta para um índice de aproximadamente $6 \%$ de enfermeiros com ideações suicidas..$^{35,50-51}$

Apesar das inúmeras situações que culminam em repercussões à saúde mental dos enfermeiros atuantes no enfrentamento à Covid-19, é possível reconhecer a existência de fatores protetivos como o apoio social e familiar, desenvolvimento da espiritualidade e da resiliência, diminuem a chance de adoecimento mental em até seis vezes. ${ }^{26}$

A capacidade de superação e reinvenção do profissional de enfermagem ${ }^{52}$ não substitui as necessárias intervenções ocupacionais em saúde mental que possam proporcionar o apoio social neste momento de crise, ainda que seja de maneira virtual..$^{53}$

Não obstante a isso, deve-se acentuar as limitações do presente estudo, que estão relacionadas ao elevado fluxo de informações sobre o início do momento pandêmico e um hiato investigativo ao final do segundo semestre, principalmente no contexto latino-americano.

\section{Conclusão}

Faz-se evidente, portanto, que a saúde mental dos profissionais de enfermagem encontra-se em estado de fragilidade no contexto de pandemia da Covid-19. Essa emergência na saúde mundial, acabou por desencadear consequências físicas e psicológicas que possivelmente se estenderão em um futuro pós-pandêmico.

Cabe a reflexão sobre as circunstâncias que estão presentes no cotidiano desses agentes, tais como jornada de trabalho excessiva, precarização do ambiente de trabalho causada pela má gestão governamental, negligência do autocuidado e deficiências no apoio social. Desse modo, fica nítida a indispensabilidade da conscientização sobre os problemas que põe em risco a saúde mental dos enfermeiros operantes em pandemias.

Aponta-se ainda a necessidade de realizações de novas investigações sobre a temática, evidenciando as estratégias de apoio à saúde mental dos profissionais atuantes no enfretamento à Covid-19, bem como as repercussões tardias, visto que o contexto de pandemia se estende e suas 
13 | Faria MGA, França KCFG, Guedes FC, Soares MS, Gallasch CH, Alves LVV

consequências ainda são imprecisas.

\section{Referências}

1. Pan American Health Organization (PAHO); World Health Organization (WHO). WHO Characterizes COVID-19 as a pandemic [Internet]? World Health Organization; 2020 [cited 2020 Jun 15]. Available from: https:/www.paho.org/hq/index.php?option=com_content\&view=article\&id=15756:who-characterizes-covid19-as-a-pandemic\&Itemid=1926\&lang=en

2. Ministry of Health (BR). Coronavirus: Brazil. https://covid.saude.gov.br/2020

3. Nguyen LH, Drew DA, Joshi AD, Guo CG, Ma W, Mehta RS, et al. Risk of Covid-19 among frontline healthcare workers and the general community: a prospective cohort study. Lancet Public Health. 2020 Sept;5(9):e475-83. doi: https://doi.org/10.1016/S2468-2667(20)30164-X

4. Zhan M, Qin Y, Xue X, Zhu S. Death from COVID-19 of 23 health care workers in China. N Engl J Med. 2020;382(23):2267-8. doi: https://doi.org/10.1056/NEJMc2005696.

5. Federal Nursing Council (COFEN). Professionals infected with Covid-19 informed by the health service [Internet]. Brasília (DF): Cofen; 2020 [access in 2020 Jun 26]. Available in: http://observatoriodaenfermagem.cofen.gov.br/

6. Silva MP, Bernardo MH, Souza HA. Relationship between mental health and work: the conception of trade unionists and possible ways of coping. Rev Bras Health Ocup. 2016;41:e23. doi: https://doi.org/10.1590/23176369000003416

7. Grincenkov FR. Hospital and health psychology in coping with coronavirus: need and proposal for action. REV HU 2020;46:1-2. doi: https://doi.org/10.34019/1982-8047.2020.v46.30050

8. Mendes KDS, Silveira RCCP, Galvão CM. Integrative review: research method for the incorporation of evidence in health and nursing. Text Context Sick. 2008;17(4):758-64. doi: https://doi.org/10.1590/S010407072008000400018

9. Whittemore R, Knafl K. The integrative review: updated methodology. J Adv Nurs. 2005;52(5):546-53. doi: https://doi.org/10.1111/j.1365-2648.2005.03621.x

10. Santos CMC, Pepper CAM, Noble MRC. The PICO strategy for the construction of the research question and search for evidence. Rev Latinoam Sick. 2007;15(3):1-4. doi: https://doi.org/10.1590/S010411692007000300023

11. Ouzzani M, Hammady H, Fedorowicz Z, Elmagarmid A. Rayyan-a web and mobile app for systematic reviews. Syst Rev. 2016;5(1):1-10. doi: https://doi.org/10.1186/s13643-016-0384-4

12. Zhang X, Jiang X, Ni P, Li H, Li C, Zhou Q, et al. Association between resilience and burnout of front-line nurses at the peak of the COVID-19 pandemic: positive and negative affect as mediators in Wuhan. Int J Ment Health Nurs. 2021; Online ahead of print. doi: https://doi.org/10.1111/inm.12847

13. Çınar D, Kılıç Akça N, Zorba Bahçeli P, Bağ Y. Perceived stress and affecting factors related to COVID19 pandemic of emergency nurses in Turkey. J Nurs Manag. 2021; Online ahead of print. doi: 
https://doi.org/10.1111/jonm.13329

14. Simonetti V, Durante A, Ambrosca R, Arcadi P, Graziano G, Pucciarelli G, et al. Anxiety, sleep disorders and self-efficacy among nurses during COVID-19 pandemic: a large cross-sectional study. J Clin Nurs. 2021;30(9-10):1360-71. doi: https://dx.doi.org/10.1111\%2Fjocn.15685

15. Yurtseven Ş, Arslan S. Anxiety levels of university hospital nurses during the Covid-19 pandemic. Perspect Psychiatr Care. 2021; Online ahead of print. doi: https://doi.org/10.1111/ppc.12719

16. Havaei F, Ma A, Staempfli S, MacPhee M. Nurses' workplace conditions impacting their mental health during covid-19: a cross-sectional survey study. Healthcare (Basel). 2021;9(1):84. doi: https://dx.doi.org/10.3390\%2Fhealthcare9010084

17. Sagherian K, Steege LM, Cobb SJ, Cho H. Insomnia, fatigue and psychosocial well-being during COVID19 pandemic: a cross-sectional survey of hospital nursing staff in the United States. J Clin Nurs. 2020; Online ahead of print. doi: https://dx.doi.org/10.1111\%2Fjocn.15566

18. Vitale E, Galatola V, Mea R. Exploring within and between gender differences in burnout levels in Italian nurses engaged in the COVID-19 health emergency: a cohort observational study. Minerva Psichiatr. 2020;61(4):162-70. doi: https://doi.org/10.23736/S0391-1772.20.02090-7

19. Chen R, Sun C, Chen JJ, Jen HJ, Kang XL, Kao CC, et al. A large-scale survey on trauma, burnout, and posttraumatic growth among nurses during the COVID-19 pandemic. Int J Ment Health Nurs. 2021;30(1):10216. doi: https://doi.org/10.1111/inm.12796

20. Labrague LJ, De los Santos JAA. COVID-19 anxiety among front-line nurses: predictive role of organisational support, personal resilience and social support. J Nurs Manag. 2020;28(7):1653-61. doi: https://doi.org/10.1111/jonm.13121

21. Saricam M. COVID-19-related anxiety in nurses working on front lines in Turkey. Nurs Midwifery Stud. 2020;9(3):178-81. doi: https://doi.org/10.4103/nms.nms_40_20

22. Murat M, Köse S, Savaşer S. Determination of stress, depression and burnout levels of front-line nurses during the COVID-19 pandemic. Int J Ment Health Nurs. 2021;30(2):533-43. doi: https://doi.org/10.1111/inm.12818

23. Zhan Y, Liu Y, Liu H, Li M, Shen Y, Gui L, et al. Factors associated with insomnia among Chinese frontline nurses fighting against COVID-19 in Wuhan: a cross-sectional survey. J Nurs Manag. 2020;28(7):1525-35. doi: https://doi.org/10.1111/jonm.13094

24. Dal'Bosco EB, Floriano LSM, Skupien SV, Arcaro G, Martins AR, Anselmo ACC. Mental health of nursing in coping with COVID-19 at a regional university hospital. Rev Bras Enferm. 2020;73(Supl 2):e20200434. doi: https://doi.org/10.1590/0034-7167-2020-0434

25. Sampaio F, Sequeira C, Teixeira L. Nurses' mental health during the Covid-19 outbreak: a cross-sectional study. J Occup Environ Med. 2020;62(10):783-7. doi: https://doi.org/10.1097/jom.0000000000001987

26. Kim SC, Quiban C, Sloan C, Montejano A. Predictors of poor mental health among nurses during COVID-19 pandemic. Nurs Open. 2021;8( 2):900-7. doi: https://doi.org/10.1002/nop2.697

27. Bahadir-Yilmaz E, Yüksel A. State anxiety levels of nurses providing care to patients with COVID-19 in 
Turkey. Perspect Psychiatr Care. 2020; Online ahead of print. doi: https://doi.org/10.1111/ppc.12661

28. Crowe S, Howard AF, Vanderspank-Wright B, Gillis P, McLeod F, Penner C, et al. The effect of COVID19 pandemic on the mental health of Canadian critical care nurses providing patient care during the early phase pandemic: a mixed method study. Intensive Crit Care Nurs. 2021;63:102999. doi: https://doi.org/10.1016/j.iccn.2020.102999

29. Mekonen E, Shetie B, Muluneh N. The psychological impact of COVID-19 outbreak on nurses working in the northwest of Amhara regional state referral hospitals, northwest Ethiopia. Psychol Res Behav Manag. 2020;13:1353-64. doi: https://doi.org/10.2147/PRBM.S291446

30. The Joanna Briggs Institute. Joanna Briggs Institute reviewer's manual. Adelaide (Australia): The Joanna Briggs Institute; 2014.

31. Cai Z, Zheng S, Huang Y, Zhang X, Qiu Z, Huang A, et al. Emotional and cognitive responses and behavioral coping of Chinese medical workers and general population during the pandemic of COVID-19. Int J Environ Res Public Health. 2020;17(17):6198. doi: https://dx.doi.org/10.3390/ijerph17176198

32. Díaz Tobajas MC, Juarros Ortiz N, García Martínez B, Sáez Gavilán C. Study on anxiety in intensive care nursing professionals facing the process of death. Enferm Glob. 2017;16(1):256-65. doi: http://dx.doi.org/10.6018/eglobal.16.1.232221

33. Yang S, Kwak SG, Ko EJ, Chang MC. The mental health burden of the COVID-19 pandemic on physical therapists. Int J Environ Res Public Health. 2020;17(10):3723. doi: https://dx.doi.org/10.3390/ijerph17103723

34. Fauzi MFM, Yusoff HM, Robat RM, Saruan NAM, Ismail KI, Haris AFM. Doctors' mental health in the midst of covid-19 pandemic: The role of work demands and recovery experiences. Int J Environ Res Public Health. 2020;17(19):7340. doi: https://dx.doi.org/10.3390/ijerph17197340

35. Hong S, Ai M, Xu X, Wang W, Chen J, Zhang Q, et al. Immediate psychological impact on nurses working at 42 government-designated hospitals during COVID-19 outbreak in China: a cross-sectional study. Nurs Outlook. 2021;69(1):6-12. doi: https://doi.org/10.1016/j.outlook.2020.07.007

36. Mo Y, Deng L, Zhang L, Lang Q, Pang H, Liao C, et al. Anxiety of nurses to support Wuhan in fighting against COVID-19 epidemic and its correlation with work stress and self-efficacy. J Clin Nurs. 2021;30(34):397-405. doi: https://doi.org/10.1111/jocn.15549

37. Evans-Lacko S, Knapp M. Global patterns of workplace productivity for people with depression: absenteeism and presenteeism costs across eight diverse countries. Soc Psychiatry Psychiatr Epidemiol. 2016;51(11):1525-37. doi: https://doi.org/10.1007/s00127-016-1278-4

38. Lee CM, Cadigan JM, Rhew IC. Increases in loneliness among young adults during the COVID-19 pandemic and association with increases in mental health problems. J Adolesc Health. 2020;67(5):714-7. doi: https://doi.org/10.1016/j.jadohealth.2020.08.009

39. Armitage R, Nellums LB. COVID-19 and the consequences of isolating the elderly. Lancet Public Health. 2020;5(5):e256. doi: https://doi.org/10.1016/S2468-2667(20)30061-X

40. Prado cep. Occupational stress: causes and consequences. Rev Bras Trab Med. 2016;14(3):285-9. doi: https://doi.org/10.5327/Z1679-443520163515 
41. Liu CY, Yang YZ, Zhang XM, Xu X, Dou QL, Zhang WW, et al. The prevalence and influencing factors in anxiety in medical workers fighting COVID-19 in China: a cross-sectional survey. Epidemiol Infect. 2020;148:e98. doi: https://doi.org/10.1017/S0950268820001107

42. Shen X, Zou X, Zhong X, Yan J, Li L. Psychological stress of ICU nurses in the time of COVID-19. Crit Care. 2020;24(1):200. doi: https://doi.org/10.1186/s13054-020-02926-2

43. Paiano M, Jaques AE, Nacamura PAB, Salci MA, Radovanovic T, Carreira L. Mental health of health professionals in China during pandemic of the new coronavirus: integrative review. Rev Bras Sick. 2020;73(Suppl 2):1-9. doi: https://doi.org/10.1590/0034-7167-2020-0338

44. Gutiérrez Alvarez AK, Cruz Almaguer AY, Zaldivar Santos ED. Management of psychological safety of health personnel in emergency situations due to COVID-19 in the hospital or isolation context. Rev Cubana Enferm [Internet]. 2020 [cited 2021 Ene 03];36(2):1-19. Available in: http://www.revenfermeria.sld.cu/index.php/enf/article/view/3704

45. Almeida LA, Medeiros IDS, Barros AG, Martins CCF, Santos VEP. Generating factors of Burnout Syndrome in health professionals. Rev Pesq Cuid Fundam. 2016;8( 3):4623. doi: https://dx.doi.org/10.9789/21755361.2016.v8i3.4623-4628

46. Oak DPC, Rocha LP, Barlem JGT, Dias JS, Schallenberger CD. Workloads and nursing worker health: integrative review. Cogitare Sick. 2017;22(1):1-10. doi: http://dx.doi.org/10.5380/ce.v22i1.46569

47. Moreira MM, Marcondes C, Geremia DS. Sleep patterns among nursing professionals. Rev Health Care. 2015;13(44):11-6. doi: https://doi.org/10.13037/ras.vol13n44.2493

48. Luceño-Moreno L, Talavera-Velasco B, García-Albuerne Y, Martín-García J. Symptoms of posttraumatic stress, anxiety, depression, levels of resilience and burnout in spanish health personnel during the COVID-19 pandemic. Int J Environ Res Public Health. 2020;17(5):5514. doi: https://dx.doi.org/10.3390/ijerph17155514

49. Silva DSD, Tavares NVS, Alexandre ARG, Freitas DA, Brêda MZ, Albuquerque MCS, et al. Depression and suicide risk among nursing professionals: an integrative review. Rev Esc Enferm USP. 2015;49(6):1027-36. doi: https://doi.org/10.1590/S0080-623420150000600020

50. Kapilan N. Suicides cases among nurses in India due to COVID-19 and possible prevention strategies. Asian J Psychiatr. 2020;54:102434. doi: https://dx.doi.org/10.1016/j.ajp.2020.102434

51. Reger MA, Piccirillo ML, Buchman-Schmitt JM. COVID-19, mental health, and suicide risk among health care workers: looking beyond the crisis. J Clin Psychiatry. 2020;81(5):20com13381. doi: https://dx.doi.org/10.4088/JCP.20com13381

52. Santarone K, McKenney M, Elkbuli A. Preserving mental health and resilience in frontline healthcare workers during COVID-19. Am J Emerg Med. 2020;38(7):1530-1 doi: https://doi.org/10.1016/j.ajem.2020.04.030

53. $\mathrm{Ng}$ B. Solutions to prevent and address physician burnout during the pandemic in Mexico. Indian J Psychiatry. 2020;62(Suppl 3):S5467-9. doi: https://dx.doi.org/10.4103/psychiatry.IndianJPsychiatry_840_20

Editora Científica: Cristiane Cardoso de Paula

Editora associada: Daiana Foggiato de Siqueira 
17 | Faria MGA, França KCFG, Guedes FC, Soares MS, Gallasch CH, Alves LVV

\section{Autor correspondente}

Magda Guimarães de Araujo Faria

E-mail: magda.faria@live.com

Endereço: Universidade do Estado do Rio de Janeiro, Faculdade de Enfermagem. Boulevard Vinte e Oito de Setembro 157, Vila Isabel - Rio de Janeiro, RJ - Brasil. CEP: 20551-030

\section{Contribuições de Autoria}

\section{1 - Magda Guimarães de Araujo Faria}

Concepção ou desenho do estudo/pesquisa, análise e/ou interpretação dos dados, revisão final com participação crítica e intelectual no manuscrito.

\section{2 - Kalyane Cristine Ferreira Gonçalves França}

Concepção ou desenho do estudo/pesquisa, análise e/ou interpretação dos dados, revisão final com participação crítica e intelectual no manuscrito.

\section{3 - Fernanda Costa Guedes}

Concepção ou desenho do estudo/pesquisa, análise e/ou interpretação dos dados, revisão final com participação crítica e intelectual no manuscrito.

\section{4 - Melissa dos Santos Soares}

Concepção ou desenho do estudo/pesquisa, análise e/ou interpretação dos dados, revisão final com participação crítica e intelectual no manuscrito.

\section{5 - Cristiane Helena Gallasch}

Concepção ou desenho do estudo/pesquisa, análise e/ou interpretação dos dados, revisão final com participação crítica e intelectual no manuscrito.

\section{6 - Luciana Valadão Vasconcelos Alves}

Concepção ou desenho do estudo/pesquisa, análise e/ou interpretação dos dados, revisão final com participação crítica e intelectual no manuscrito.

\section{Como citar este artigo}

Faria MGA, France KCFG, Guedes FC, Soares MS, Gallasch CH, lvv Alves. Repercussions for mental health of nursing professionals who are in the face of Covid-19: integrativereview. Rev. Sick. Ufsm. 2021 [Access in: Years Month Day]; vol.11 e70: 1-17. DOI: https://doi.org/10.5902/2179769264313 